\title{
ANALYTICAL EXPRESSIONS FOR EXOTHERMIC EXPLOSIONS IN A SLAB
}

V. Ananthaswamy**1, M. Subha ${ }^{2}$

*1 Department of Mathematics, The Madura College, Madurai-625011, Tamil Nadu, India

${ }^{2}$ Department of Mathematics, Madurai Sivakasi Nadars Pioneer Meenakshi Women's College Poovanthi, Sivagangai District, Tamil Nadu, India.

*Correspondence Author: ananthu9777@ rediffmail.com

\begin{abstract}
:
Analytical solutions for the strongly exothermic decomposition of combustible material are discussed in this paper. Combustible material uniformly distributed between symmetrically heated parallel plates under Sensitized, Arrhenius and Bimolecular reaction rates are also discussed neglecting consumption of material. Approximate analytical expressions of steady state temperature fields are derived by using Homotopy analysis method (HPM) for various values of relevant dimensionless parameters. Analytical results are compounded with perturbation technique and numerical simulation. Analytical results are coinciding with numerical simulation and agreement is noted. The present method simple, less computational and applicable for solving strongly non-linear initial and boundary value problems.
\end{abstract}

Keywords:

Thermal explosions; Kinetics reactions; Non-linear boundary value problem; Homotopy analysis method; Numerical simulation.

\section{INTRODUCTION}

Thermal ignition studies and heat transfers in slab of reactive material are useful in slab to improve design and operation of industrial and engineering devices [1-3]. One of insidious conditions is internal heat generation which affects quality of industrial product after it is cast, coated, moulded, forged or laminated. Industrial products may be discolored; warped, weakened or ignited spontaneously if heat is liberated from chemical reaction inside material is not removed or prevented. Squire [4] found that discoloration of central region of a tall stack of plywood panels was due to excessive internal heat generation from exothermic chemical reactions in curing of glue. Damage of panels can be avoided if magnitude of thermal criticality parameter was not exceeded. Thermal criticality phenomenon is an important concept in combustion theory it occurs when rate of internal heat generation exceeds rate of heat loss to cool surroundings [5,6]. Criticality conditions provide criterion for safety of storage materials capable of undergoing exothermic reaction. Investigation of boundary conditions is point of surface temperature is function of position [7,8]. This paper is to derive the approximate analytical expressions of steady state temperature fields for the Sensitized, Arrhenius and Bimolecular reaction rates.

\section{MATHEMATICAL FORMULATION OF THE PROBLEM}




\section{INTERNATIONAL JOURNAL Of RESEARCH - GRANTHAALAYAH \\ A knowledge Repository}

Science

Critical regimes evaluation problem is thought of regimes separating regions of explosive and non-explosive ways of chemical reactions is main mathematical problem of thermal explosion theory [9-11]. Classical formulation problem was introduced by Frank-Kamenetskii [11]. Equation for heat balance in original variables together with the boundary conditions can be written as:

$k \frac{d^{2} T}{d \bar{y}}+Q C_{0} A\left(\frac{K T}{v h}\right)^{m} e^{\frac{-E}{R T}}=0, \frac{d T}{d \bar{y}}(0)=0, T(a)=T_{0}$

where $T$ is the absolute temperature, $T_{0}$ the wall temperature, $k$ the thermal conductivity of the material, $Q$ the heat of reaction, $A$ the rate constant, $E$ the activation energy, $R$ the universal gas constant, $C_{0}$ the initial concentration of the reactant species, $h$ the planck's number, $K$ the Boltzmann's constant, $v$ vibration frequency, $a$ the slab half width, $\bar{y}$ is the distance measured in the normal direction to the plane and $m$ is the numerical exponent such that $m=\{-2,0,1 / 2\}$ represent the numerical exponent for Sensitised, Arrhenius and Bimolecular kinetics respectively [9-11]. Dimensionless variables is introduced in eqn.(1) are introduced as follows [11]:

$\theta=\frac{E\left(T-T_{0}\right)}{R T_{0}^{2}}, \varepsilon=\frac{R T_{0}}{E}, y=\frac{\bar{y}}{a}, \quad \lambda=\frac{Q E A a^{2} C_{0} K^{m} T_{0}^{m-2} e^{\frac{-E}{R T_{0}}}}{v^{m} h^{m} R k}$

Substituting the eqns. (2) into an eqn.(1), we obtain the non-linear differential equations as

$\frac{d^{2} \theta}{d y^{2}}+\lambda(1+\varepsilon \theta)^{m} e^{\left(\frac{\theta}{1+\varepsilon \theta}\right)}$

and the corresponding boundary conditions as

$\frac{d \theta}{d y}(0)=0, \quad \theta(1)=0$

where $\lambda, \varepsilon$ represent the Frank-Kamenetskii and activation energy parameters respectively.

\section{SOLUTION OF BOUNDARY VALUE PROBLEM USING HOMOTOPY ANALYSIS METHOD (HAM)}

Homotopy analysis method is non-perturbing analytical method for obtaining solutions in nonlinear equations and has been successfully applied to numerous problems in science and engineering [13-28]. It offers ability to adjust and control convergences of series solutions. It is most effective method for obtaining analytical solutions to highly non-linear differential equations. Previous applications of HAM have mainly focused on non-linear differential equations in which the non-linearity is a polynomial in terms of the unknown function and its derivatives.

Liao [13-21] proposed a powerful analytical method for non-linear problems, namely the Homotopy analysis method. This method provides an analytical solution in terms of an infinite power series. However, there is a practical need to evaluate this solution and to obtain numerical 


\section{INTERNATIONAL JOURNAL Of RESEARCH - GRANTHAALAYAH \\ A knowledge Repository}

Science

values from the infinite power series. In order to investigate the accuracy of the Homotopy analysis method (HAM) solution with a finite number of terms, the system of differential equations were solved. The Homotopy analysis method is a good technique comparing to another perturbation method.

Homotopy perturbation method [29-33] is a special case of Homotopy analysis method. Different from all reported perturbation and non-perturbative techniques, the Homotopy analysis method itself provides us with a convenient way to control and adjust the convergence region and rate of approximation series, when necessary. Briefly speaking, this method has the following advantages: It is valid even if a given non-linear problem does not contain any small/large parameter at all; it can be employed to efficiently approximate a non-linear problem by choosing different sets of base functions. The Homotopy analysis method contains the auxiliary parameter $h$, which provides us with a simple way to adjust and control the convergence region of solution series. Using this method, we can obtain the following solution to (1) and (2) (see Appendix B). The approximate analytical solution of the equations (1) and (2) using the Homotopy analysis method is given by

$\theta(y)=\left(\frac{\lambda\left(1-y^{2}\right)}{2}\right)-h\left[\begin{array}{l}\left(\frac{-\lambda^{2}(1+\varepsilon m)}{2}\right)\left(\frac{y^{2}}{2}-\frac{y^{4}}{12}\right)-\left(\frac{\lambda^{3} \varepsilon(1-m)}{4}\right)\left(\frac{y^{2}}{2}-\frac{y^{4}}{6}+\frac{y^{6}}{30}\right) \\ +\left(\frac{5 \lambda^{2}(1+\varepsilon m)}{24}\right)+\left(\frac{11 \lambda^{3} \varepsilon(1-m)}{120}\right)\end{array}\right]$

The approximated analytical solution of for the temperature field for Sensitized $(m=-2)$, Arrhenius $(m=0)$ and the Bimolecular reaction rates respectively are given by

$$
\begin{aligned}
& \theta(y ; \lambda, \varepsilon, m=-2)=\left(\frac{\lambda\left(1-y^{2}\right)}{2}\right)-h\left[\begin{array}{l}
\left.\left(\frac{-\lambda^{2}(1-2 \varepsilon)}{2}\right)\left(\frac{y^{2}}{2}-\frac{y^{4}}{12}\right)-\left(\frac{3 \lambda^{3} \varepsilon}{4}\right)\left(\frac{y^{2}}{2}-\frac{y^{4}}{6}+\frac{y^{6}}{30}\right)\right] \\
+\left(\frac{5 \lambda^{2}(1-2 \varepsilon)}{24}\right)+\left(\frac{11 \lambda^{3} \varepsilon}{40}\right)
\end{array}\right] \\
& \theta(y ; \lambda, \varepsilon, m=0)=\left(\frac{\lambda\left(1-y^{2}\right)}{2}\right)-h\left[\begin{array}{l}
\left.\left(\frac{-\lambda^{2}}{2}\right)\left(\frac{y^{2}}{2}-\frac{y^{4}}{12}\right)-\left(\frac{\lambda^{3} \varepsilon}{4}\right)\left(\frac{y^{2}}{2}-\frac{y^{4}}{6}+\frac{y^{6}}{30}\right)\right] \\
+\left(\frac{5 \lambda^{2}}{24}\right)+\left(\frac{11 \lambda^{3} \varepsilon}{120}\right)
\end{array}\right]-h\left[\begin{array}{l}
\left(\frac{-\lambda^{2}(2+\varepsilon)}{4}\right)\left(\frac{y^{2}}{2}-\frac{y^{4}}{12}\right)-\left(\frac{\lambda^{3} \varepsilon}{8}\right)\left(\frac{y^{2}}{2}-\frac{y^{4}}{6}+\frac{y^{6}}{30}\right) \\
+\left(\frac{5 \lambda^{2}(2+\varepsilon)}{48}\right)+\left(\frac{11 \lambda^{3} \varepsilon}{240}\right)
\end{array}\right] \\
& \theta(y ; \lambda, \varepsilon, m=1 / 2)=\left(\frac{\lambda\left(1-y^{2}\right)}{2}\right)
\end{aligned}
$$




\section{INTERNATIONAL JOURNAL Of RESEARCH - GRANTHAALAYAH

\section{a. PREVIOUS WORK (PERTURBATION AND MULTIVARIATE SERIES SUMMATION TECHNIQUES [8]])}

The analytical solution for the temperature field for Sensitized, Arrhenius and Bimolecular reactions rates are given by

$$
\begin{aligned}
& \theta(y ; \lambda, \varepsilon, m=-2)=\left(\frac{-\lambda\left(y^{2}-1\right)}{2}\right)-\left(\frac{\lambda^{2}\left(y^{2}-1\right)\left(y^{2}-5\right)(2 \varepsilon-1)}{24}\right)+\left(\frac{\lambda^{3}}{360}\right)\left(y^{2}-1\right) \\
& \theta(y ; \lambda, \varepsilon, m=0)=\left(\frac{-\lambda\left(y^{2}-1\right)}{2}\right)+\left(\frac{\lambda^{2}\left(y^{2}-1\right)\left(y^{2}-5\right)}{24}\right) \\
&+\left(\frac{\lambda^{3}}{360}\right)\left(y^{2}-1\right)\left(-2 y^{4}+3 y^{4} \varepsilon-12 \varepsilon y^{2}+13 y^{2}+33 \varepsilon-47\right)+O\left(\lambda^{4}\right) \\
& \theta(y ; \lambda, \varepsilon, m=1 / 2)=\left(\frac{-\lambda\left(y^{2}-1\right)}{2}\right)-\left(\frac{\lambda^{2}\left(y^{2}-1\right)\left(y^{2}-5\right)(2+\varepsilon)}{48}\right)+\left(\frac{\lambda^{3}}{1440}\right)\left(y^{2}-1\right) \\
&\left(4 y^{4} \varepsilon-8 y^{4}+y^{4} \varepsilon^{2}+4 \varepsilon y^{2}+221 \varepsilon^{2}+52 y^{2}+y^{2} \varepsilon^{2}-14 \varepsilon^{2}-56 \varepsilon-188\right)+O\left(\lambda^{4}\right)
\end{aligned}
$$

\section{NUMERICAL SIMULATION}

To find the accuracy of our analytical method, the non-linear differential eqns. (3) and (4) are also solved by numerical methods. The function bvp4c in Matlab/Scilab software which is a function of solving non-linear boundary value problems (BVPs) for ordinary differential equations are used to solve these equations numerically. Our analytical results are compared with numerical simulations and it gives a good agreement (See Figures: 1-3). The Matlab/Scilab program is also given in Appendix $\mathrm{C}$.

\section{RESULTS AND DISCUSSION}

Figure 1-3 shows dimensionless temperature fields $\theta(y)$ for Sensitized, Arrhenius and Bimolecular reaction rates versus the dimensionless distance $y$ respectively. From these Figs. it is clear that when the Frank-Kamenetskii parameter $\lambda$ increases, the corresponding temperature fields also increases to some fixed values of the activation energy parameter $\varepsilon$. Also, explosion in bimolecular reaction seems to occur faster than in Arrhenius and Sensitized reactions. Table 1 shows error table in present work, previous work and numerical simulation for various kinetics ( $m=-2, m=0, m=0.5)$. This table shows average error percentage between the present work and numerical simulation are very small compared to average error percentage between previous work and numerical simulation for Sensitized and Bimolecular reaction rates. 


\section{INTERNATIONAL JOURNAL Of RESEARCH - GRANTHAALAYAH \\ A knowledge Repository}

Science

\section{CONCLUSION}

Steady state solution for strongly exothermic decomposition of combustible material uniformly distributed between symmetrically heated parallel plates under Sensitized, Arrhenius and Bimolecular reaction rates are derived. Approximate analytical solutions for dimensionless temperature profile for various kinetics are derived by using the Homotopy analysis method. Primary result of this work was simple. This analytical results and numerical simulation show good agreement. This method is extremely simple it solves all other non-linear boundary initial and boundary value problems also.

\section{APPENDIX: A \\ BASIC CONCEPT OF HOMOTOPY ANALYSIS METHOD}

Consider the following differential equation [13-19]:

$N[u(t)]=0$

Where $N$ is a nonlinear operator, $t$ denotes an independent variable, $u(t)$ is an unknown function. For simplicity, we ignore all boundary or initial conditions, which can be treated in the similar way. By means of generalizing the conventional Homotopy method, Liao [12] constructed the so-called zero-order deformation equation as:

$(1-p) L\left[\varphi(t ; p)-u_{0}(t)\right]=p h H(t) N[\varphi(t ; p)]$

where $p \in[0,1]$ is the embedding parameter, $h \neq 0$ is a nonzero auxiliary parameter, $H(t) \neq 0$ is an auxiliary function, $L$ an auxiliary linear operator, $u_{0}(t)$ is an initial guess of $u(t)$, $\varphi(t: p)$ is an unknown function. It is important, that one has great freedom to choose auxiliary unknowns in HAM. Obviously, when $p=0$ and $p=1$, it holds:

$\varphi(t ; 0)=u_{0}(t)$ and $\varphi(t ; 1)=u(t)$

respectively. Thus, as $p$ increases from 0 to 1 , the solution $\varphi(t ; p)$ varies from the initial guess $u_{0}(t)$ to the solution $u(t)$. Expanding $\varphi(t ; p)$ in Taylor series with respect to $p$, we have:

$\varphi(t ; p)=u_{0}(t)+\sum_{m=1}^{+\infty} u_{m}(t) p^{m}$

where

$u_{m}(t)=\left.\frac{1}{m !} \frac{\partial^{m} \varphi(t ; p)}{\partial p^{m}}\right|_{p=0}$

If the auxiliary linear operator, the initial guess, the auxiliary parameter $h$, and the auxiliary function are so properly chosen, the series (A.4) converges at $p=1$ then we have:

$u(t)=u_{0}(t)+\sum_{m=1}^{+\infty} u_{m}(t)$.

Differentiating (A.2) for $m$ times with respect to the embedding parameter $p$, and then setting 


\section{INTERNATIONAL JOURNAL Of RESEARCH - GRANTHAALAYAH

$p=0$ and finally dividing them by $m !$, we will have the so-called $m-t h$ order deformation equation as:

$$
L\left[u_{m}-\chi_{m} u_{m-1}\right]=h H(t) \mathfrak{R}_{m}\left(\vec{u}_{m-1}\right)
$$

where

$$
\mathfrak{R}_{m}\left(\vec{u}_{m-1}\right)=\frac{1}{(m-1) !} \frac{\partial^{m-1} N[\varphi(t ; p)]}{\partial p^{m-1}}
$$

and

$\chi_{m}=\left\{\begin{array}{l}0, m \leq 1, \\ 1, m>1 .\end{array}\right.$

Applying $L^{-1}$ on both side of equation (A7), we get

$u_{m}(t)=\chi_{m} u_{m-1}(t)+h L^{-1}\left[H(t) \Re_{m}\left(u_{m-1}\right)\right]$

In this way, it is easily to obtain $u_{m}$ for $m \geq 1$, at $M^{\text {th }}$ order, we have

$$
u(t)=\sum_{m=0}^{M} u_{m}(t)
$$

When $M \rightarrow+\infty$, we get an accurate approximation of the original equation (A.1). For the convergence of the above method we refer the reader to Liao [9]. If equation (A.1) admits unique solution, then this method will produce the unique solution.

\section{APPENDIX: B}

ANALYTICAL EXPRESSIONS OF THE NON-LINEAR DIFFERENTIAL EQNS. (3)-(4) FOR VARIOUS KINETICS USING THE HOMOTOPY ANALYSIS METHOD [13-28]

In this Appendix, we indicate how the eqn. (5) is derived in this paper. When $(1+\varepsilon \theta)$ is small then the eqn.(3) reduces to

$$
\frac{d^{2} \theta}{d y^{2}}+\lambda+\lambda(1+m \varepsilon) y+\lambda \varepsilon(1-m) y^{2}=0
$$

We construct the Homotopy as follows:

$$
(1-p)\left[\frac{d^{2} \theta}{d y^{2}}+\lambda\right]=h p\left[\frac{d^{2} \theta}{d y^{2}}+\lambda+\lambda(1+m \varepsilon) y+\lambda \varepsilon(1-m) y^{2}\right]
$$

The approximate solution of the eqn.(B.2) is, $\theta=\theta_{0}+p \theta_{1}+p^{2} \theta_{2}+$

Substituting (B.3) in (B.2) we have 


\section{INTERNATIONAL JOURNAL Of RESEARCH - GRANTHAALAYAH

$$
(1-p)\left[\frac{d^{2}\left(\theta_{0}+p \theta_{1}+p^{2} \theta_{2}+\ldots . .\right)}{d y^{2}}+\lambda\right]=h p\left[\begin{array}{l}
\frac{d^{2}\left(\theta_{0}+p \theta_{1}+p^{2} \theta_{2}+\ldots . .\right)}{d y^{2}}+\lambda \\
+\lambda(1+m \varepsilon)\left(\theta_{0}+p \theta_{1}+p^{2} \theta_{2}+\ldots . .\right) \\
+\lambda \varepsilon(1-m)\left(\theta_{0}+p \theta_{1}+p^{2} \theta_{2}+\ldots . .\right)^{2}
\end{array}\right]
$$

Comparing the coefficients of like powers of $p$ in (B.5) we get

$$
\begin{aligned}
& p^{0}: \frac{d^{2} \theta_{0}}{d y^{2}}+\lambda=0 \\
& p^{1}: \frac{d^{2} \theta_{1}}{d y^{2}}-(h+1)\left(\frac{d^{2} \theta_{0}}{d y^{2}}+\lambda\right)-h \lambda(1+m \varepsilon) \theta_{0}-\lambda \varepsilon(1-m) \theta_{0}^{2}=0
\end{aligned}
$$

The initial approximations are as follows

$\theta_{0}^{\prime}(0)=0$ and $\theta_{0}(1)=0$

$\theta_{i}^{\prime}(0)=0$ and $\theta_{i}(1)=0 \quad i=1,2,3 \ldots \ldots$

Solving the eqns.(B.5) and (B.6) and using the boundary conditions (B.7) and (B.8) we can obtain the following results:

$$
\begin{aligned}
& \theta_{0}=\left(\frac{\lambda\left(1-y^{2}\right)}{2}\right) \\
& \theta_{1}=-h\left[\begin{array}{l}
\left.\left(\frac{-\lambda^{2}(1+\varepsilon m)}{2}\right)\left(\frac{y^{2}}{2}-\frac{y^{4}}{12}\right)-\left(\frac{\lambda^{3} \varepsilon(1-m)}{4}\right)\left(\frac{y^{2}}{2}-\frac{y^{4}}{6}+\frac{y^{6}}{30}\right)+\left(\frac{5 \lambda^{2}(1+\varepsilon m)}{24}\right)\right] \\
+\left(\frac{11 \lambda^{3} \varepsilon(1-m)}{120}\right)
\end{array}\right]
\end{aligned}
$$

According to the HAM, we can conclude that

$$
\theta=\lim _{p \rightarrow 1} \theta(y)=\theta_{0}+\theta_{1}
$$

After putting the eqns.(B.9) and (B.10) into an eqn.(B.11), we obtain the solution in the text eqn. (5)

\section{APPENDIX C:}

MATLAB/SCILAB PROGRAM TO FIND THE NUMERICAL SOLUTION OF THE NON-LINEAR DIFFERENTIAL EQNS. (3) AND (4)

function pdex 4

$\mathrm{m}=0$;

$\mathrm{x}=$ linspace $(0,1)$;

$\mathrm{t}=$ linspace $(0,10000)$;

sol=pdepe $(\mathrm{m}, @$ pdex4pde, $@$ pdex4ic, @ pdex4bc,x,t $)$; 


\section{INTERNATIONAL JOURNAL Of RESEARCH - GRANTHAALAYAH

$\mathrm{u} 1=\operatorname{sol}(:,:, 1)$

figure

$\operatorname{plot}(\mathrm{x}, \mathrm{u} 1(\mathrm{end}, \mathrm{)}))$

title('u1 $(\mathrm{x}, \mathrm{t})$ ')

xlabel('Distance x')

ylabel('u1(x,2)')

$\%$

function $[\mathrm{c}, \mathrm{f}, \mathrm{s}]=\operatorname{pdex} 4 \mathrm{pde}(\mathrm{x}, \mathrm{t}, \mathrm{u}, \mathrm{DuDx})$

$\mathrm{c}=1$;

$\mathrm{f}=\mathrm{DuDx}$

$\mathrm{a}=.5 ; \mathrm{e}=.1 ; \mathrm{M}=0$;

$\mathrm{F}=\mathrm{a}^{*}\left(\left(1+\mathrm{e}^{*} \mathrm{u}\right)^{\wedge} \mathrm{M}\right) * \exp \left(\mathrm{u} /\left(1+\mathrm{e}^{*} \mathrm{u}\right)\right)$;

$\mathrm{s}=\mathrm{F}$;

$\%$

function $\mathrm{u} 0=\operatorname{pdex} 4 \mathrm{ic}(\mathrm{x}) ; \quad \%$ create a initial conditions

$\mathrm{u} 0=1$

$\%$

function[pl,ql,pr,qr]=pdex4bc(xl,ul,xr,ur,t) \%create a boundary conditions

$\mathrm{pl}=0$;

$\mathrm{ql}=1$;

pr $=$ ur- 0

$\mathrm{qr}=0$;

\section{APPENDIX D: \\ DETERMINING THE REGION OF H FOR VALIDITY}

The analytical solution should converge. It should be noted that the auxiliary parameter $h$ controls the convergence and accuracy of the solution series. The analytical solution represented by eqn.(5) contains the auxiliary parameter $h$, which gives the convergence region and rate of approximation for the Homotopy analysis method. The valid region of $h$ is about (-1.2 to -1$)$, when $m=-2, \varepsilon=0.1$ and $\lambda=0.4$. Similarly, we can find the value of the convergence control parameter $h$ for different values of the constant parameters.

\section{APPENDIX E:}

\section{NOMENCLATURE}

\begin{tabular}{|c|l|}
\hline Symbols & \multicolumn{1}{|c|}{ Meaning } \\
\hline$T$ & Absolute temperature \\
\hline$T_{0}$ & Wall temperature \\
\hline$k$ & Thermal conductivity of the material \\
\hline$Q$ & Heat of reaction \\
\hline
\end{tabular}




\section{INTERNATIONAL JOURNAL Of RESEARCH - GRANTHAALAYAH

\begin{tabular}{|l|l|}
\hline$A$ & Rate constant \\
\hline$E$ & Activation energy \\
\hline$R$ & Universal gas constant \\
\hline$C_{0}$ & Initial concentration of the reactant species \\
\hline$h$ & Planck's number \\
\hline$K$ & Boltzmann's constant \\
\hline$v$ & Vibration frequency \\
\hline$a$ & Slab half width \\
\hline $\bar{y}$ & Distance measured in the normal direction to the plane \\
\hline$m$ & Numerical exponent \\
\hline$\theta$ & Dimensionless temperature field \\
\hline$y$ & Dimensionless distance \\
\hline$\lambda$ & Frank-Kamenetskii parameter \\
\hline$\varepsilon$ & Activation energy parameter \\
\hline
\end{tabular}

Table 1: Error table for present work, previous work and numerical simulation

\begin{tabular}{|c|c|c|c|c|c|}
\hline \multicolumn{6}{|c|}{$m=-2, \lambda=0.1, \varepsilon=0.1$} \\
\hline$x$ & $\begin{array}{l}\text { (a) Present } \\
\text { work }\end{array}$ & $\begin{array}{l}\text { (b) Previous } \\
\text { work }\end{array}$ & $\begin{array}{l}\text { (c) Numerical } \\
\text { solution }\end{array}$ & $\begin{array}{l}\text { Error \% } \\
\text { (a) \& (c) }\end{array}$ & $\begin{array}{c}\text { Error \% } \\
(b) \&(c)\end{array}$ \\
\hline 0 & 0.05170 & 0.05159 & 0.05172 & 0.03867 & 0.2514 \\
\hline 0.2 & 0.04961 & 0.04952 & 0.04963 & 0.04030 & 0.2216 \\
\hline 0.4 & 0.04338 & 0.04329 & 0.04339 & 0.02305 & 0.2305 \\
\hline 0.6 & 0.03301 & 0.03295 & 0.03302 & 0.03028 & 0.2112 \\
\hline 0.8 & 0.01853 & 0.01850 & 0.01854 & 0.05394 & 0.2158 \\
\hline 1 & 0 & 0 & 0 & 0 & 0 \\
\hline \multicolumn{4}{|c|}{ Average error \% } & 0.03104 & 0.1884 \\
\hline \multicolumn{6}{|c|}{$m=-2, \lambda=0.2, \varepsilon=0.1$} \\
\hline$x$ & $\begin{array}{l}\text { (a) Present } \\
\text { work }\end{array}$ & $\begin{array}{l}\text { (b) Previous } \\
\text { work }\end{array}$ & $\begin{array}{l}\text { (c) Numerical } \\
\text { solution }\end{array}$ & $\begin{array}{l}\text { Error \% } \\
(a) \&(c)\end{array}$ & $\begin{array}{l}\text { Error \% } \\
(b) \&(c)\end{array}$ \\
\hline 0 & 0.1069 & 0.1061 & 0.1072 & 0.2798 & 1.0261 \\
\hline 0.2 & 0.1026 & 0.1018 & 0.1029 & 0.2916 & 1.0690 \\
\hline 0.4 & 0.08961 & 0.08893 & 0.08985 & 0.2671 & 1.0239 \\
\hline 0.6 & 0.06808 & 0.06761 & 0.06827 & 0.2783 & 0.9668 \\
\hline 0.8 & 0.03816 & 0.03791 & 0.03825 & 0.2353 & 0.8889 \\
\hline 1 & 0 & 0 & 0 & 0 & 0 \\
\hline \multicolumn{4}{|c|}{ Average error \% } & 0.2254 & 0.8291 \\
\hline \multicolumn{6}{|c|}{$m=-2, \lambda=0.3, \varepsilon=0.1$} \\
\hline$x$ & $\begin{array}{l}\text { (a) Present } \\
\text { work }\end{array}$ & $\begin{array}{l}\text { (b) Previous } \\
\text { work }\end{array}$ & $\begin{array}{c}\text { (c) Numerical } \\
\text { solution }\end{array}$ & $\begin{array}{c}\text { Error \% } \\
(a) \&(c)\end{array}$ & $\begin{array}{l}\text { Error \% } \\
(b) \&(c)\end{array}$ \\
\hline 0 & 0.1658 & 0.1630 & 0.1671 & 0.7779 & 2.4536 \\
\hline
\end{tabular}




\section{INTERNATIONAL JOURNAL Of RESEARCH - GRANTHAALAYAH \\ A knowledge Repository}

Science

\begin{tabular}{|c|c|c|c|c|c|}
\hline 0.2 & 0.1593 & 0.1564 & 0.1603 & 0.6238 & 2.4329 \\
\hline 0.4 & 0.1389 & 0.1366 & 0.1399 & 0.7148 & 2.3588 \\
\hline 0.6 & 0.1055 & 0.1037 & 0.1061 & 0.5655 & 2.2620 \\
\hline 0.8 & 0.05892 & 0.05808 & 0.0593 & 0.6401 & 2.0573 \\
\hline 1 & 0 & 0 & 0 & 0 & 0 \\
\hline \multicolumn{4}{|c|}{ Average error \% } & 0.5537 & 1.9274 \\
\hline \multicolumn{6}{|c|}{$m=-2, \lambda=0.4, \varepsilon=0.1$} \\
\hline$x$ & $\begin{array}{l}\text { (a) Present } \\
\text { work }\end{array}$ & $\begin{array}{l}\text { (b) Previous } \\
\text { work }\end{array}$ & $\begin{array}{l}\text { (c) Numerical } \\
\text { solution }\end{array}$ & $\begin{array}{l}\text { Error \% } \\
\text { (a)\&(c) }\end{array}$ & $\begin{array}{l}\text { Error \% } \\
\text { (b)\&(c) }\end{array}$ \\
\hline 0 & 0.2284 & 0.2218 & 0.2321 & 1.5941 & 4.4377 \\
\hline 0.2 & 0.2191 & 0.2128 & 0.2226 & 1.5723 & 4.4025 \\
\hline 0.4 & 0.1911 & 0.1858 & 0.1941 & 1.5456 & 4.2762 \\
\hline 0.6 & 0.1448 & 0.1410 & 0.1470 & 1.4966 & 4.0816 \\
\hline 0.8 & 0.08090 & 0.07888 & 0.08201 & 1.3535 & 3.8166 \\
\hline 1 & 0 & 0 & 0 & 0 & 0 \\
\hline \multicolumn{4}{|c|}{ Average error \% } & 1.2604 & 3.5028 \\
\hline \multicolumn{6}{|c|}{$m=-2, \lambda=0.5, \varepsilon=0.1$} \\
\hline$x$ & $\begin{array}{c}\text { (a) Present } \\
\text { work }\end{array}$ & $\begin{array}{c}\text { (b) Previous } \\
\text { work }\end{array}$ & $\begin{array}{c}\text { (c) Numerical } \\
\text { solution }\end{array}$ & $\begin{array}{c}\text { Error \% } \\
(a) \&(c)\end{array}$ & $\begin{array}{l}\text { Error \% } \\
(b) \&(c)\end{array}$ \\
\hline 0 & 0.2951 & 0.2823 & 0.3034 & 2.7357 & 6.9545 \\
\hline 0.2 & 0.2829 & 0.2707 & 0.2908 & 2.7166 & 6.9120 \\
\hline 0.4 & 0.2466 & 0.2363 & 0.2533 & 2.6451 & 6.7114 \\
\hline 0.6 & 0.1869 & 0.1792 & 0.1915 & 2.4021 & 6.4230 \\
\hline 0.8 & 0.1041 & 0.1002 & 0.1066 & 2.3452 & 6.0038 \\
\hline 1 & 0 & 0 & 0 & 0 & 0 \\
\hline \multicolumn{4}{|c|}{ Average error \% } & 2.14085 & 5.5008 \\
\hline \multicolumn{6}{|c|}{$m=0, \lambda=0.1, \varepsilon=0.1$} \\
\hline$x$ & $\begin{array}{l}\text { (a) Present } \\
\text { work }\end{array}$ & $\begin{array}{l}\text { (b) Previous } \\
\text { work }\end{array}$ & $\begin{array}{l}\text { (c) Numerical } \\
\text { solution }\end{array}$ & $\begin{array}{l}\text { Error \% } \\
(a) \&(c)\end{array}$ & $\begin{array}{l}\text { Error \% } \\
(b) \&(c)\end{array}$ \\
\hline 0 & 0.05210 & 0.05220 & 0.05218 & 0.1533 & 0.0383 \\
\hline 0.2 & 0.05000 & 0.05010 & 0.05007 & 0.1398 & 0.0599 \\
\hline 0.4 & 0.04370 & 0.04379 & 0.04377 & 0.1599 & 0.0465 \\
\hline 0.6 & 0.03324 & 0.03331 & 0.03329 & 0.1502 & 0.0601 \\
\hline 0.8 & 0.01866 & 0.01869 & 0.01868 & 0.1071 & 0.0534 \\
\hline 1 & 0 & 0 & 0 & 0 & 0 \\
\hline \multicolumn{4}{|c|}{ Average error \% } & 0.1184 & 0.0430 \\
\hline \multicolumn{6}{|c|}{$m=0, \lambda=0.2, \varepsilon=0.1$} \\
\hline$x$ & $\begin{array}{l}\text { (a) Present } \\
\text { work }\end{array}$ & $\begin{array}{c}\text { (b) Previous } \\
\text { work }\end{array}$ & $\begin{array}{l}\text { (c) Numerical } \\
\text { solution }\end{array}$ & $\begin{array}{l}\text { Error \% } \\
(a) \&(c)\end{array}$ & $\begin{array}{l}\text { Error \% } \\
(b) \&(c)\end{array}$ \\
\hline 0 & 0.1084 & 0.1093 & 0.1093 & 0.8234 & 0.0000 \\
\hline
\end{tabular}




\section{INTERNATIONAL JOURNAL Of RESEARCH - GRANTHAALAYAH \\ A knowledge Repository}

Science

\begin{tabular}{|c|c|c|c|c|c|}
\hline 0.2 & 0.1040 & 0.1049 & 0.1048 & 0.7634 & 0.0954 \\
\hline 0.4 & 0.0909 & 0.09156 & 0.09154 & 0.6995 & 0.0218 \\
\hline 0.6 & 0.0690 & 0.06950 & 0.06950 & 0.7194 & 0.0000 \\
\hline 0.8 & 0.03864 & 0.03891 & 0.03890 & 0.6684 & 0.0257 \\
\hline 1 & 0 & 0 & 0 & 0 & 0 \\
\hline \multicolumn{4}{|c|}{ Average error \% } & 0.6124 & 0.0239 \\
\hline \multicolumn{6}{|c|}{$m=0, \lambda=0.3, \varepsilon=0.1$} \\
\hline$x$ & $\begin{array}{l}\text { (a) Present } \\
\text { work }\end{array}$ & $\begin{array}{l}\text { (b) Previous } \\
\text { work }\end{array}$ & $\begin{array}{l}\text { (c) Numerical } \\
\text { solution }\end{array}$ & $\begin{array}{l}\text { Error \% } \\
\text { (a)\&(c) }\end{array}$ & $\begin{array}{l}\text { Error \% } \\
\text { (b)\&(c) }\end{array}$ \\
\hline 0 & 0.1701 & 0.1720 & 0.1724 & 1.3341 & 0.2320 \\
\hline 0.2 & 0.1639 & 0.1650 & 0.1654 & 0.9069 & 0.2418 \\
\hline 0.4 & 0.1421 & 0.1439 & 0.1442 & 1.4563 & 0.2081 \\
\hline 0.6 & 0.1080 & 0.1090 & 0.1093 & 1.1894 & 0.2745 \\
\hline 0.8 & 0.0603 & 0.06088 & 0.06099 & 1.1313 & 0.1804 \\
\hline 1 & 0 & 0 & 0 & 0 & 0 \\
\hline \multicolumn{4}{|c|}{ Average error \% } & 1.0030 & 0.1895 \\
\hline \multicolumn{6}{|c|}{$m=0, \lambda=0.4, \varepsilon=0.1$} \\
\hline$x$ & $\begin{array}{c}\text { (a) Present } \\
\text { work }\end{array}$ & $\begin{array}{l}\text { (b) Previous } \\
\text { work }\end{array}$ & $\begin{array}{c}\text { (c) Numerical } \\
\text { solution }\end{array}$ & $\begin{array}{c}\text { Error \% } \\
(a) \&(c)\end{array}$ & $\begin{array}{l}\text { Error \% } \\
(b) \&(c)\end{array}$ \\
\hline 0 & 0.2411 & 0.2411 & 0.2432 & 0.8635 & 0.8635 \\
\hline 0.2 & 0.2311 & 0.2311 & 0.2331 & 0.8580 & 0.8580 \\
\hline 0.4 & 0.2014 & 0.2014 & 0.2030 & 0.7882 & 0.7881 \\
\hline 0.6 & 0.1524 & 0.1523 & 0.1535 & 0.7166 & 0.7818 \\
\hline 0.8 & 0.0849 & 0.0848 & 0.08541 & 0.5971 & 0.7142 \\
\hline 1 & 0 & 0 & 0 & 0 & 0 \\
\hline \multicolumn{4}{|c|}{ Average error \% } & 0.6372 & 0.6676 \\
\hline \multicolumn{6}{|c|}{$m=0, \lambda=0.5, \varepsilon=0.1$} \\
\hline$x$ & $\begin{array}{l}\text { (a) Present } \\
\text { work }\end{array}$ & $\begin{array}{l}\text { (b) Previous } \\
\text { work }\end{array}$ & $\begin{array}{l}\text { (c) Numerical } \\
\text { solution }\end{array}$ & $\begin{array}{l}\text { Error \% } \\
(a) \&(c)\end{array}$ & $\begin{array}{l}\text { Error \% } \\
(b) \&(c)\end{array}$ \\
\hline 0 & 0.3192 & 0.3172 & 0.3238 & 1.4206 & 2.0383 \\
\hline 0.2 & 0.3059 & 0.3040 & 0.3102 & 1.3863 & 1.9987 \\
\hline 0.4 & 0.2662 & 0.2646 & 0.2697 & 1.2977 & 1.8901 \\
\hline 0.6 & 0.2011 & 0.1994 & 0.2034 & 1.1308 & 1.9666 \\
\hline 0.8 & 0.1117 & 0.1110 & 0.1129 & 1.0629 & 1.6830 \\
\hline 1 & 0 & 0 & 0 & 0 & 0 \\
\hline \multicolumn{4}{|c|}{ Average error \% } & 1.0498 & 1.5961 \\
\hline \multicolumn{6}{|c|}{$m=0.5, \lambda=0.1, \varepsilon=0.1$} \\
\hline$x$ & $\begin{array}{c}\text { (a) Present } \\
\text { work }\end{array}$ & $\begin{array}{l}\text { (b) Previous } \\
\text { work }\end{array}$ & $\begin{array}{l}\text { (c) Numerical } \\
\text { solution }\end{array}$ & $\begin{array}{c}\text { Error \% } \\
(a) \&(c)\end{array}$ & $\begin{array}{l}\text { Error \% } \\
(b) \&(c)\end{array}$ \\
\hline 0 & 0.05220 & 0.04781 & 0.05229 & 0.1721 & 8.5676 \\
\hline 0.2 & 0.05009 & 0.04694 & 0.05018 & 0.1794 & 6.4567 \\
\hline
\end{tabular}




\section{INTERNATIONAL JOURNAL Of RESEARCH - GRANTHAALAYAH \\ A knowledge Repository}

Science

\begin{tabular}{|c|c|c|c|c|c|}
\hline 0.4 & 0.04378 & 0.04715 & 0.04386 & 0.1824 & 7.5011 \\
\hline 0.6 & 0.03330 & 0.04761 & 0.03336 & 0.1799 & 42.758 \\
\hline 0.8 & 0.01869 & 0.03822 & 0.01872 & 0.1603 & 104.1666 \\
\hline 1 & 0 & 0 & 0 & 0 & 0 \\
\hline \multicolumn{4}{|c|}{ Average error \% } & 0.1460 & 28.2417 \\
\hline \multicolumn{6}{|c|}{$m=0.5, \lambda=0.2, \varepsilon=0.1$} \\
\hline$x$ & $\begin{array}{l}\text { (a) Present } \\
\text { work }\end{array}$ & $\begin{array}{l}\text { (b) Previous } \\
\text { work }\end{array}$ & $\begin{array}{l}\text { (c) Numerical } \\
\text { solution }\end{array}$ & $\begin{array}{l}\text { Error \% } \\
(a) \&(c)\end{array}$ & $\begin{array}{c}\text { Error \% } \\
\text { (b)\&(c) }\end{array}$ \\
\hline 0 & 0.1088 & 0.09125 & 0.1098 & 0.9107 & 16.8943 \\
\hline 0.2 & 0.1044 & 0.08869 & 0.1054 & 0.9488 & 15.8539 \\
\hline 0.4 & 0.09115 & 0.08381 & 0.09198 & 0.9041 & 8.8824 \\
\hline 0.6 & 0.06922 & 0.07569 & 0.06981 & 0.8452 & 8.4229 \\
\hline 0.8 & 0.03876 & 0.05416 & 0.03906 & 0.7680 & 38.6584 \\
\hline 1 & 0 & 0 & 0 & 0 & 0 \\
\hline \multicolumn{4}{|c|}{ Average error \% } & 0.7295 & 14.7854 \\
\hline \multicolumn{6}{|c|}{$m=0.5, \lambda=0.3, \varepsilon=0.1$} \\
\hline$x$ & $\begin{array}{l}\text { (a) Present } \\
\text { work }\end{array}$ & $\begin{array}{l}\text { (b) Previous } \\
\text { work }\end{array}$ & $\begin{array}{l}\text { (c) Numerical } \\
\text { solution }\end{array}$ & $\begin{array}{l}\text { Error \% } \\
(a) \&(c)\end{array}$ & $\begin{array}{c}\text { Error \% } \\
(b) \&(c)\end{array}$ \\
\hline 0 & 0.1698 & 0.1303 & 0.1739 & 2.3577 & 25.0718 \\
\hline 0.2 & 0.1629 & 0.1263 & 0.1667 & 2.2795 & 24.2351 \\
\hline 0.4 & 0.1422 & 0.1170 & 0.1454 & 2.2008 & 19.5323 \\
\hline 0.6 & 0.1078 & 0.1012 & 0.1101 & 2.0890 & 8.0836 \\
\hline 0.8 & 0.0620 & 0.06872 & 0.06143 & 2.0022 & 11.8677 \\
\hline 1 & 0 & 0 & 0 & 0 & 0 \\
\hline \multicolumn{4}{|c|}{ Average error \% } & 1.8215 & 14.7984 \\
\hline \multicolumn{6}{|c|}{$m=0.5, \lambda=0.4, \varepsilon=0.1$} \\
\hline$x$ & $\begin{array}{l}\text { (a) Present } \\
\text { work }\end{array}$ & $\begin{array}{l}\text { (b) Previous } \\
\text { work }\end{array}$ & $\begin{array}{l}\text { (c) Numerical } \\
\text { solution }\end{array}$ & $\begin{array}{l}\text { Error \% } \\
(a) \&(c)\end{array}$ & $\begin{array}{c}\text { Error \% } \\
(b) \&(c)\end{array}$ \\
\hline 0 & 0.2427 & 0.1650 & 0.2462 & 1.4216 & 32.9813 \\
\hline 0.2 & 0.2327 & 0.1597 & 0.2360 & 1.3983 & 32.3305 \\
\hline 0.4 & 0.2028 & 0.1465 & 0.2054 & 1.2658 & 28.6758 \\
\hline 0.6 & 0.1534 & 0.1241 & 0.1552 & 1.1598 & 20.0387 \\
\hline 0.8 & 0.08540 & 0.08192 & 0.08634 & 1.0887 & 5.1193 \\
\hline 1 & 0 & 0 & 0 & 0 & 0 \\
\hline \multicolumn{4}{|c|}{ Average error \% } & 1.0557 & 19.8471 \\
\hline \multicolumn{6}{|c|}{$m=0.5, \lambda=0.5, \varepsilon=0.1$} \\
\hline$x$ & $\begin{array}{c}\text { (a) Present } \\
\text { work }\end{array}$ & $\begin{array}{l}\text { (b) Previous } \\
\text { work }\end{array}$ & $\begin{array}{c}\text { (c) Numerical } \\
\text { solution }\end{array}$ & $\begin{array}{c}\text { Error \% } \\
(a) \&(c)\end{array}$ & $\begin{array}{c}\text { Error \% } \\
(b) \&(c)\end{array}$ \\
\hline 0 & 0.3220 & 0.1953 & 0.3296 & 2.3058 & 40.7466 \\
\hline 0.2 & 0.3085 & 0.1889 & 0.3158 & 2.3116 & 40.1837 \\
\hline 0.4 & 0.2685 & 0.1726 & 0.2745 & 2.1858 & 37.1220 \\
\hline
\end{tabular}




\section{INTERNATIONAL JOURNAL Of RESEARCH - GRANTHAALAYAH \\ A knowledge Repository}

Science

\begin{tabular}{|c|c|c|c|c|c|}
\hline 0.6 & 0.2043 & 0.1445 & 0.2069 & 1.2566 & 30.1595 \\
\hline 0.8 & 0.1125 & 0.0938 & 0.1146 & 1.8325 & 18.1501 \\
\hline 1 & 0 & 0 & 0 & 0 & 0 \\
\hline \multicolumn{7}{|c|}{ Average error \% } & 1.6487 & 27.7270 \\
\hline
\end{tabular}

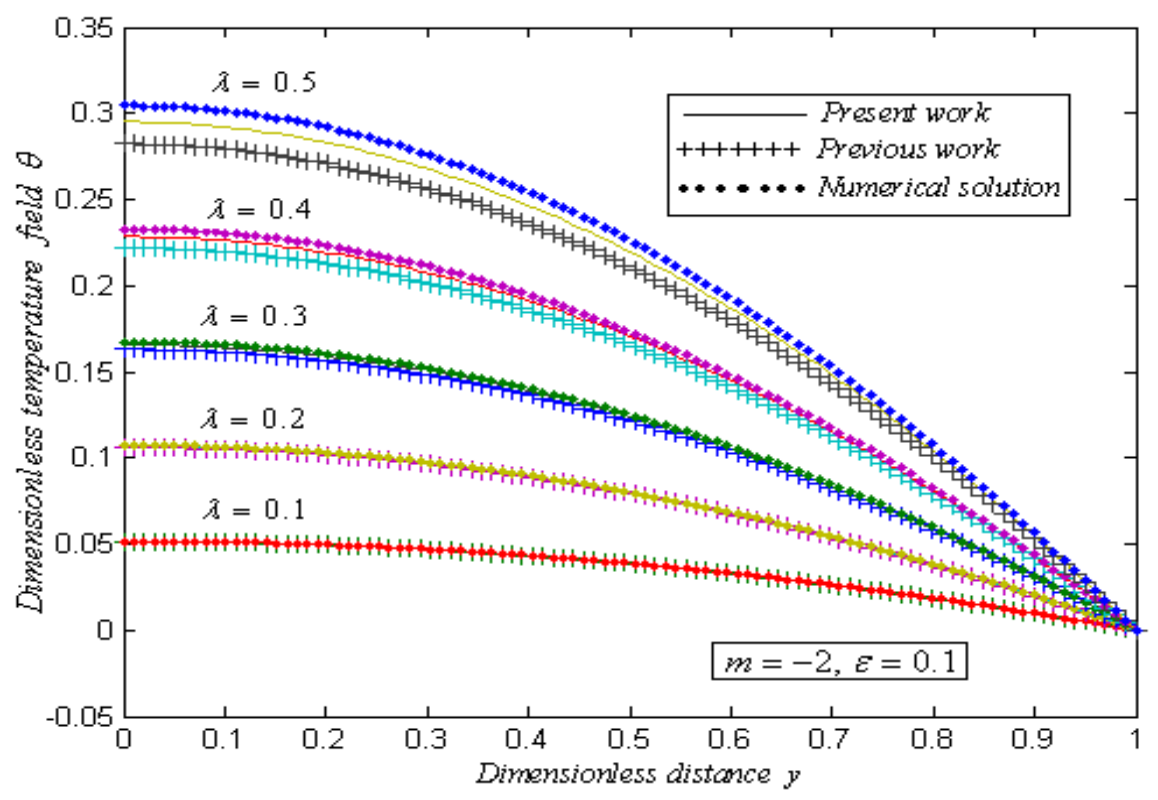

Figure.1: Dimensionless temperature filed $\theta(y)$ versus the dimensionless distance $y$. The dimensionless temperature field were computed using the eqn. (5a) (Sensitized) for various values of the dimensionless parameter $\lambda$ and when $h=-1.1$. 


\section{INTERNATIONAL JOURNAL Of RESEARCH - GRANTHAALAYAH \\ A knowledge Repository}

Science

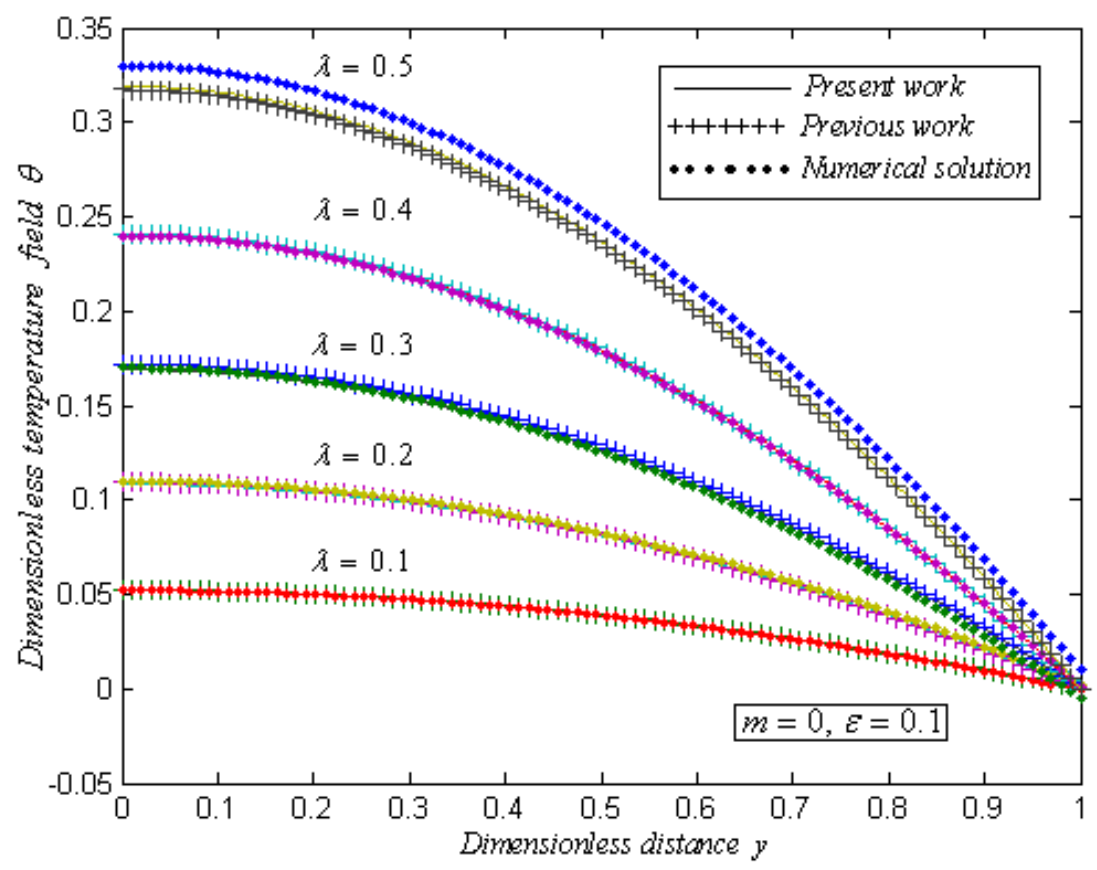

Figure.2: Dimensionless temperature filed $\theta(y)$ versus the dimensionless distance $y$. The dimensionless temperature field were computed using the eqn. (5b) (Arrhenius) for various values of the dimensionless parameter $\lambda$ and when $h=-1.1$.

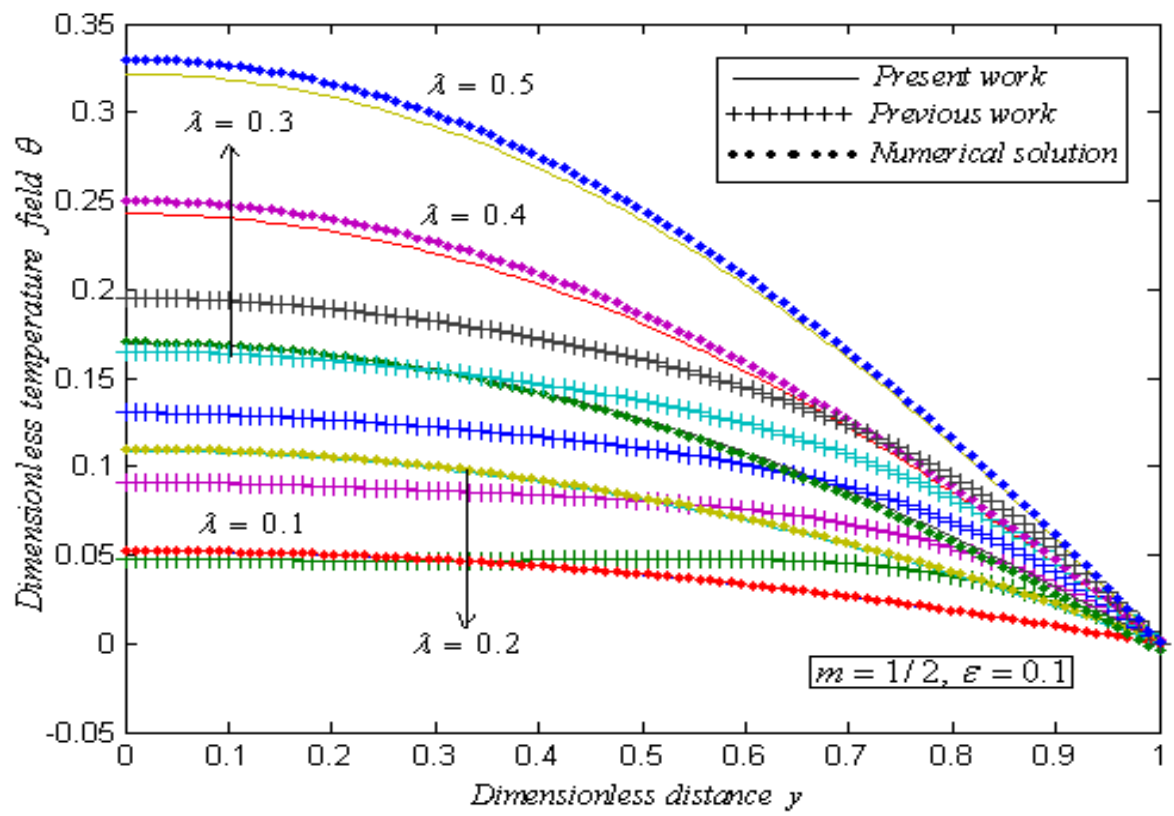

Figure.3: Dimensionless temperature filed $\theta(y)$ versus the dimensionless distance $y$. The dimensionless temperature field were computed using the eqn. (5c) (Bimolecular) for various values of the dimensionless parameter $\lambda$ and when $h=-1.1$. 


\section{INTERNATIONAL JOURNAL Of RESEARCH - GRANTHAALAYAH

\section{REFERENCES}

[1] O.D. Makinde, Hermite-Pade approach thermal stability of reacting masses in a slab with asymmetric convective cooling, Journal of the Franklin Institute 349, 2012, 957-965.

[2] L.D. Landau, E.M. Lifshitz, in: Fluid Mechanics, Addison-Wesley, Reading, Massachusetts, 191, 1959.

[3] J. Bebernes, D. Eberly, in: Mathematical Problems from Combustion Theory, SpringerVerlag, New York, 1989.

[4] W. Squire, A mathematical analysis of self-ignition, in: B. Noble (Ed.), Application of Undergraduate Mathematics in Engineering, MacMillan, New York, 18, 1967.

[5] D.A. Frank Kamenetskii, in: Diffusion and Heat Transfer in chemical kinetics, Plenum Press, New York, 1969.

[6] F. Alhama, A. Campo, The connection between the distributed and lumped models for asymmetric cooling of long slabs by heat convection, International Communications in Heat and Mass Transfer 28 (1) (2001) 127-137.

[7] M.B. Zaturska, W.H.H. Banks, Thermal explosion with variable thermal conductivity: criticality and its disappearance, IMA Journal of Applied Mathematics 34 (1985) 73-82.

[8] O.D. Makinde, Exothermic explosions in a Slab: A case study of series summation technique, International Communications in Heat and Mass Transfer 31 (8) (2004) 1227-1231.

[9] O. D. Makinde, Exothermic explosions in a slab: A case study of series summation technique,

[10] J. Bebernes, D. Eberly, Mathematical problems from combustion theory, Springer-Verlag, New York, 1989.

[11] D. A. Frank Kamenetskii, Diffusion and Heat Transfer in chemical kinetics, Plenum Press, New York 1969.

[12] O. D. Makinde, Hermite-Pade_approach to thermal stability of reacting masses in a slab with asymmetric convective cooling, Journal of the Franklin Institute 349, 2012, 957-965.

[13] S. J. Liao, The proposed Homotopy analysis technique for the solution of non-linear problems, Ph.D. Thesis, Shanghai Jiao Tong University, 1992.

[14] S. J. Liao, An approximate solution technique which does not depend upon small parameters: a special example, Int. J. Non-Linear Mech. 30, 1995, 371-380.

[15] S. J. Liao, Beyond perturbation introduction to the Homotopy analysis method, $1^{\text {st }}$ edn., Chapman and Hall, CRC press, Boca Raton ,336, 2003.

[16] S. J. Liao, On the Homotopy analysis method for nonlinear problems, Appl. Math. Comput. 147, 2004, 499-513.

[17] S. J. Liao, An optimal Homotopy-analysis approach for strongly nonlinear differential equations, Commun. Nonlinear Sci. Numer. Simulat. 15, 2010, 2003-2016.

[18] S. J. Liao, The Homotopy analysis method in non linear differential equations, Springer and Higher education press, 2012.

[19] S. J. Liao, An explicit totally analytic approximation of Blasius viscous flow problems. Int J Nonlinear Mech;34, 1999, 759-778.

[20] S. J. Liao, On the analytic solution of magneto hydrodynamic flows non-Newtonian fluids over a stretching sheet. J Fluid Mech: 488, 2003, 189-212. 


\section{INTERNATIONAL JOURNAL Of RESEARCH - GRANTHAALAYAH \\ A knowledge Repository}

Science

[21] S. J. Liao, A new branch of boundary layer flows over a permeable stretching plate. Int J Nonlinear Mech: 42, 2007, 19-30.

[22] G. Domairry, H. Bararnia, An approximation of the analytical solution of some nonlinear heat transfer equations: a survey by using Homotopy analysis method, Adv. Studies Theor. Phys. 2, 2008, 507-518.

[23] Y. Tan , H. Xu ,S. J. Liao, Explicit series solution of travelling waves with a front of Fisher equation. Chaos Solitons Fractals: 31, 2007, 462-472.

[24] S. Abbasbandy, Soliton solutions for the FitzhughNagumo equation with the homotopy analysis method. Appl Math Model: 32, 2008, 2706-2714.

[25] J. Cheng J, S. J. Liao, R. N. Mohapatra, K. Vajravelu, Series solutions of nano boundary layer flows by means of the Homotopy analysis method. J Math Anal Appl: 343, 2008, 233245.

[26] T. Hayat, Z. Abbas, Heat transfer analysis on MHD flow of a second grade fluid in a channel with porous medium. Chaos Solitons Fractals: 38,2008, 556-567.

[27] T. Hayat, R. Naz, M. Sajid, On the Homotopy solution for Poiseuille flow of a fourth grade fluid. Commun Nonlinear Sci Numer Simul: 15, 2010, 581-589.

[28] H. Jafari, C. Chun, S. M. Saeidy, Analytical solution for nonlinear gas dynamic Homotopy analysis method, Appl. Math. 4, 2009, 149-154.

[29] M. S. H. Chowdhury, I. Hashim, solutions of time-dependent Emden-Fowler type equations by Homotopy perturbation method, Phys. Lett. A., 368, 305-313, 2007.

[30] Q. K. Ghori, M. Ahmed, A. M. Siddiqui, Application of Homotopy perturbation method to squeezing flow of a Newtonian fluid, Int. J. Nonlinear. Sci. Numer.Simulat., 8(2), 2007, 179184.

[31] J. M. Coyle, J. E. Flaherty, and R. Ludwig. On the stability of mesh equidistribution strategies for time-dependent partial differential equations. J. Comput.Phys.62, 1986, 26- 39.

[32] T. Ozis, A. Yildirim, Relation of a Nonlinear Oscillator with Discontinuities, Int. J. Non-linear. Sci. Numer. Simulat., 8(2), 2007, 243-24.

[33] N. Madden and M. Stynes. A uniformly convergent numerical method for a coupled system of two singularly perturbed linear reaction-diffusion problems. IMA J. Numer.Anal., 23(4), 2003, 627-644. 